\title{
Investigation of the Aerodynamics of an Innovative Vertical-Axis Wind Turbine
}

\author{
Krzysztof Tesch ${ }^{1} \cdot$ Katarzyna Kludzinska $^{1}$. \\ Piotr Doerffer ${ }^{2}$
}

Received: 20 October 2014 / Accepted: 30 April 2015 / Published online: 15 May 2015

(C) Springer Science+Business Media Dordrecht 2015

\begin{abstract}
This paper presents the results of an experimental investigation and a three dimensional numerical analysis of the transient aerodynamic phenomena occurring in the innovative modification of classic Savonius wind turbine. An attempt to explain the increased efficiency of the innovative design a comparison with the traditional solution is undertaken. A vorticity measure based on the integral of the velocity gradient tensor second invariant is proposed in order to evaluate and compare designs. The discussed criterion is related to the vortex structures and energy dissipation. These structures are generated by the rotor and may affect the efficiency.
\end{abstract}

Keywords Wind turbines · Vorticity measures · CFD

\section{Introduction}

The object of the analysis is the modernisation of classic design of the turbine (i.e. the Savonius wind turbine), see Fig. 1. This innovative design [5] is equipped with a stator (Fig. 2) which experimentally shows increased efficiency in comparison with standard design. This is true for wind tunnel experiments at least. Both turbines have the simplest design of all devices converting wind into other energy forms, which provides opportunity to decrease its price. The original Savonius wind turbine has numerous advantages such as low noise, simplicity of design, applicability for a wide range of wind velocities. It is also independent of

Krzysztof Tesch

krzyte@pg.gda.pl

1 Fluid Mechanics Department,

Gdansk University of Technology,

ul. G. Narutowicza 11/12, 80-233 Gdansk, Poland

2 Institute of Fluid Flow Machinery,

ul. Fiszera 14, 80-231 Gdansk, Poland 
Fig. 1 Classic designs

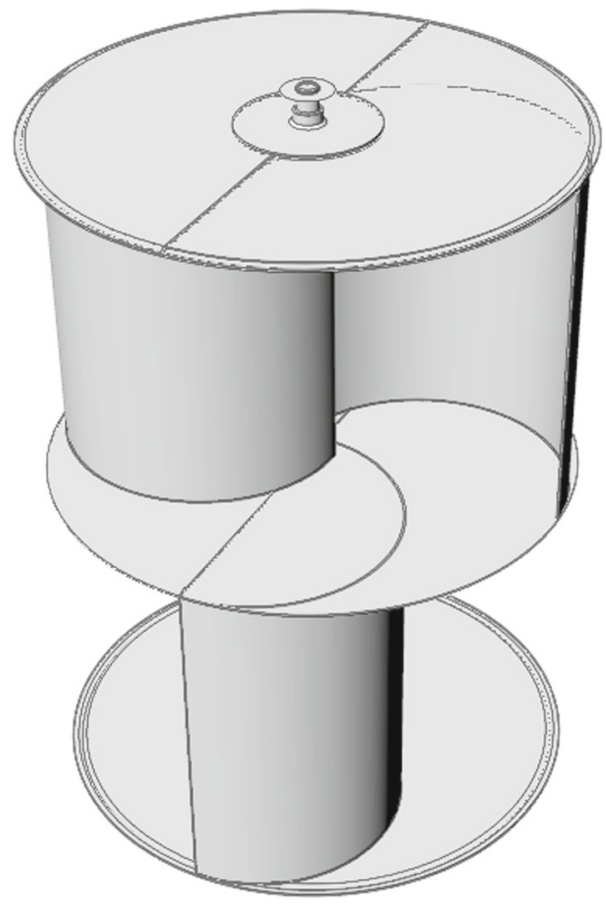

the wind direction. The biggest problem of the classic design is its relatively low efficiency. The innovative design, discussed here, is devoid of this disadvantage still being independent of the wind direction.

Wind turbine research, such as Savonius turbine and its modifications, are in line with the general strategy of development of the EU power industry. This strategy tends to quantitative increase the use of renewable energy sources. One of the limits, as far as the wind energy is concerned, it is high price of wind turbines available. The obtained results may increase the knowledge of the flow around a whole family of different types of rotors whose principle of operation is based primarily on the use of drag accompanied with a small share of lift forces. The knowledge gained during the simulations will enable more effective designs characterised by increased efficiency and operational reliability.

The flow inside the rotor is complicated and that is why in past investigations were limited only to laboratory tests $[2,3,7,14]$. Another experimental method is flow visualisation $[6,12]$, which allows capturing flow patterns characteristics for selected instantaneous positions of rotor blades with respect to the wind direction. Recently, due to the rapid development of computer hardware and software, attempts were made to analyse structure of the flow through the Savonius rotor numerically $[10,15]$. This also includes the vortex method $[1,13]$. Generally, all data presented on the numerical aspect are mostly two-dimensional. Also, papers on attempts to modify the shape of the Savonius rotor are available [7,11]. The best shapes of blade tips for a given rotor geometry and physical conditions are investigated in $[8,9,16]$.

The results of an experimental investigation of the innovative modification of classic Savonius wind turbine are presented in Section 2 together with the distributions of the non-dimensional quantities that can be easily compared with other studies. For comparison 


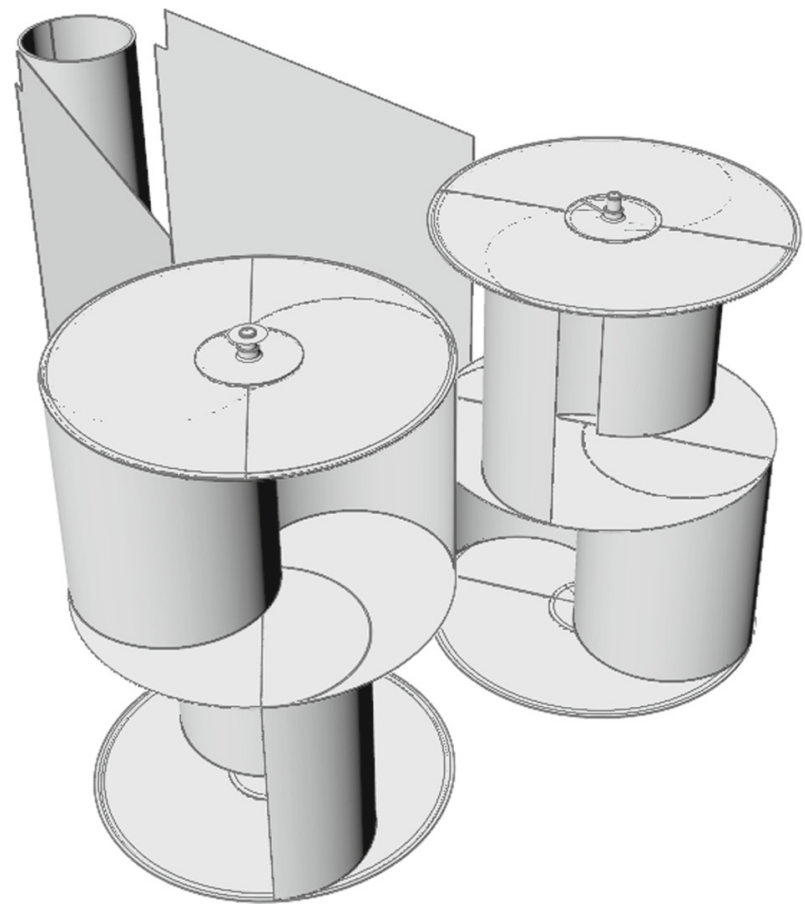

Fig. 2 Innovative designs

purposes, measurements for the Savonius design are included. The details of computations together with evaluation and comparison criteria are given in Section 3. This section also presents results of numerical calculations in order to compare different designs. Finally, conclusions are summarised in Section 4.

\section{Coefficients and Experimental Results}

\subsection{Coefficients}

Among many characteristics of the wind turbines the most important is the torque coefficient. It is commonly defined as

$$
C_{T}=\frac{T}{\frac{1}{4} \rho U^{2} D^{2} H}
$$

The above definition is valid for both the steady-state and transient flows. For the latter case one should use the time dependent torque $T(t)$ instead of $T$, meaning that in real case we deal with the distribution of $C_{T}$ as a function of the angular position of the rotor $\alpha$. The torque coefficient (1) is directly related to the efficiency of the rotor.

A typical definition of the efficiency (power coefficient) for the steady-state case takes under consideration the wind power $P_{w}=\dot{m} e_{k}=\rho U S 2^{-1} U^{2}$ and the power of the rotor 


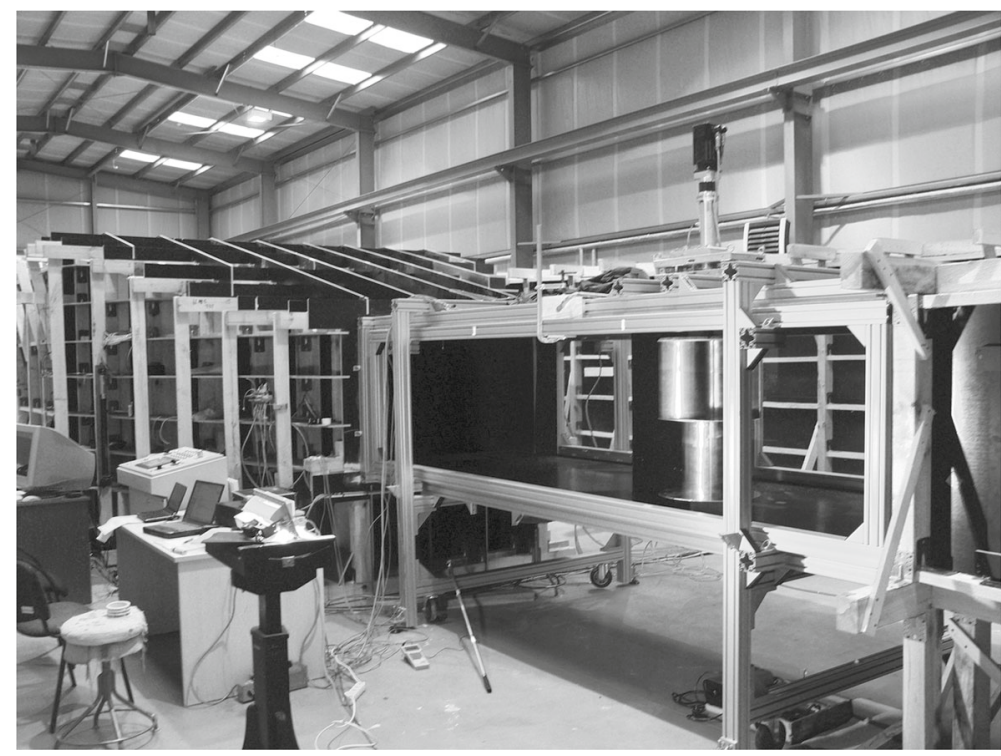

Fig. 3 Test stand

$P=\omega T$. The wind power is regarded here as the reference power. From the two above definitions we arrive at the following definition of the power coefficient

$$
C_{P}=\frac{\omega T}{\frac{1}{2} \rho U^{3} H D} .
$$

In the above definition $\omega$ is the angular velocity, $\rho$ represents the density, and $U-$ the reference velocity. $D$ is the diameter of the rotor, and $H$ is the rotor's height. For the transient case, which is typical for the Savonius rotor operation, one should consider the total energy of the wind within the time interval $\Delta t$ rather than the instantaneous power. This means that the definition (2) takes the following form now

$$
C_{P}=\frac{\omega \int_{t}^{t+\Delta t} T(t) \mathrm{d} t}{\frac{1}{2} \rho U^{3} H D \Delta t}=\frac{\omega \bar{T}}{\frac{1}{2} \rho U^{3} H D} .
$$

where $\Delta t$ stands for the time of interest (e.g. one revolution). Assuming that the time step of the transient CFD calculations is constant we can approximate the integral in Eq. 3 in the following way $\bar{T}=n^{-1} \sum_{i=1}^{n} T_{i}$ where $\bar{T}$ represents the arithmetical average. The total number of time steps is denoted here as $n$. The last definition (3) has an analogical form as the definition (2) and is the basis for comparison the innovative design with the traditional solution. Furthermore, Eqs. 1-3 are only valid for Savonius and H-Darrieus rotors. This is because the projected area is not $H D$ for horizontal-axis wind turbines.

Typically, the torque and power coefficients are expressed as a function of the rotor tip speed ratio $\lambda$ which is formally defined as the ratio between the tangential speed of the tip of a blade and the velocity of the wind $U$

$$
\lambda=\frac{\omega D}{2 U} .
$$



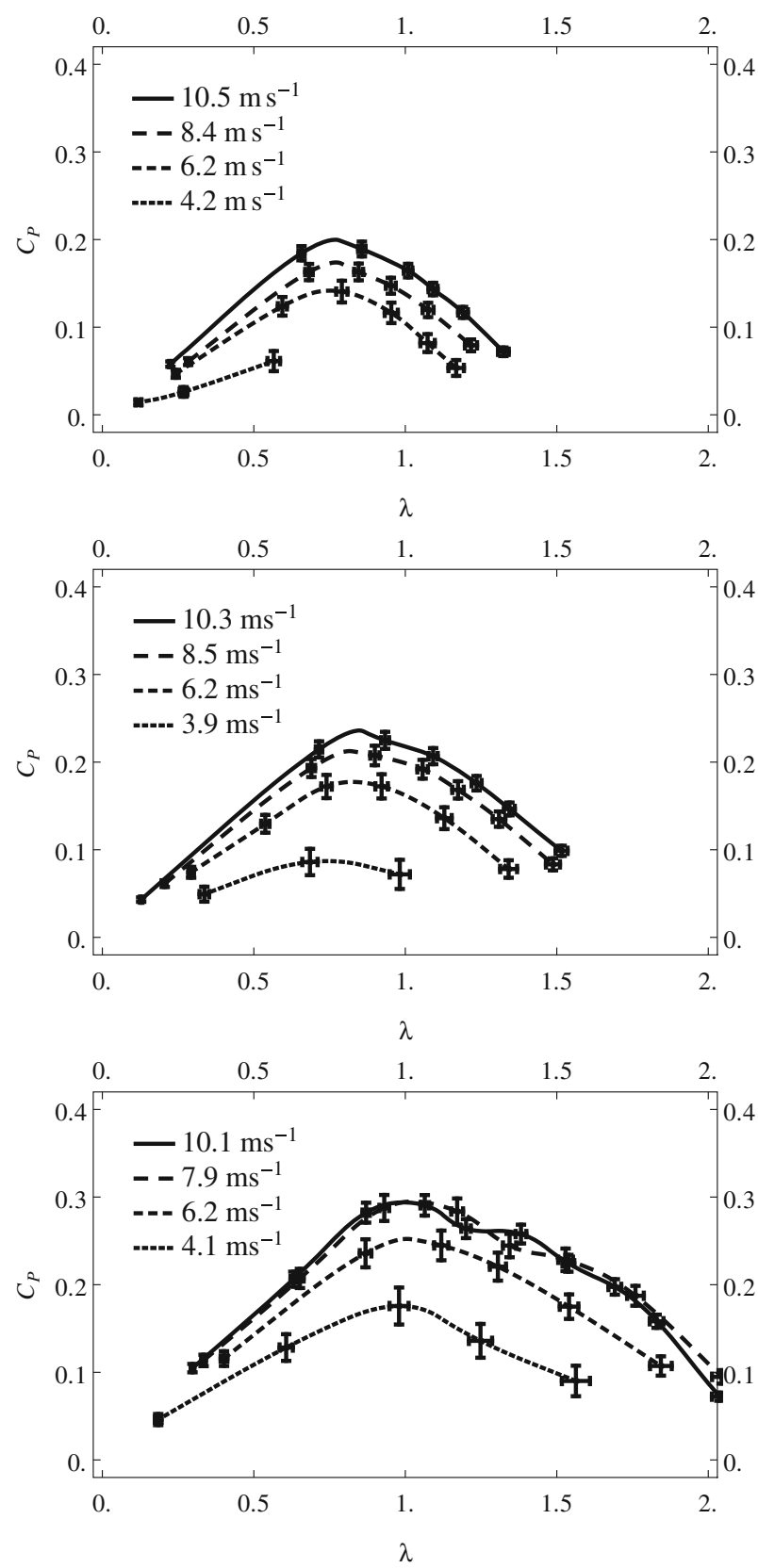

Fig. $4 C_{P}$ distribution as a function of $\lambda$ (Savonius - top, $20^{\circ}-$ middle and $40^{\circ}-$ bottom)

The non-dimensional quantities $\left(C_{P}, C_{T}\right)$ distributions as a function of $\lambda$ can now be easily compared with other studies. 

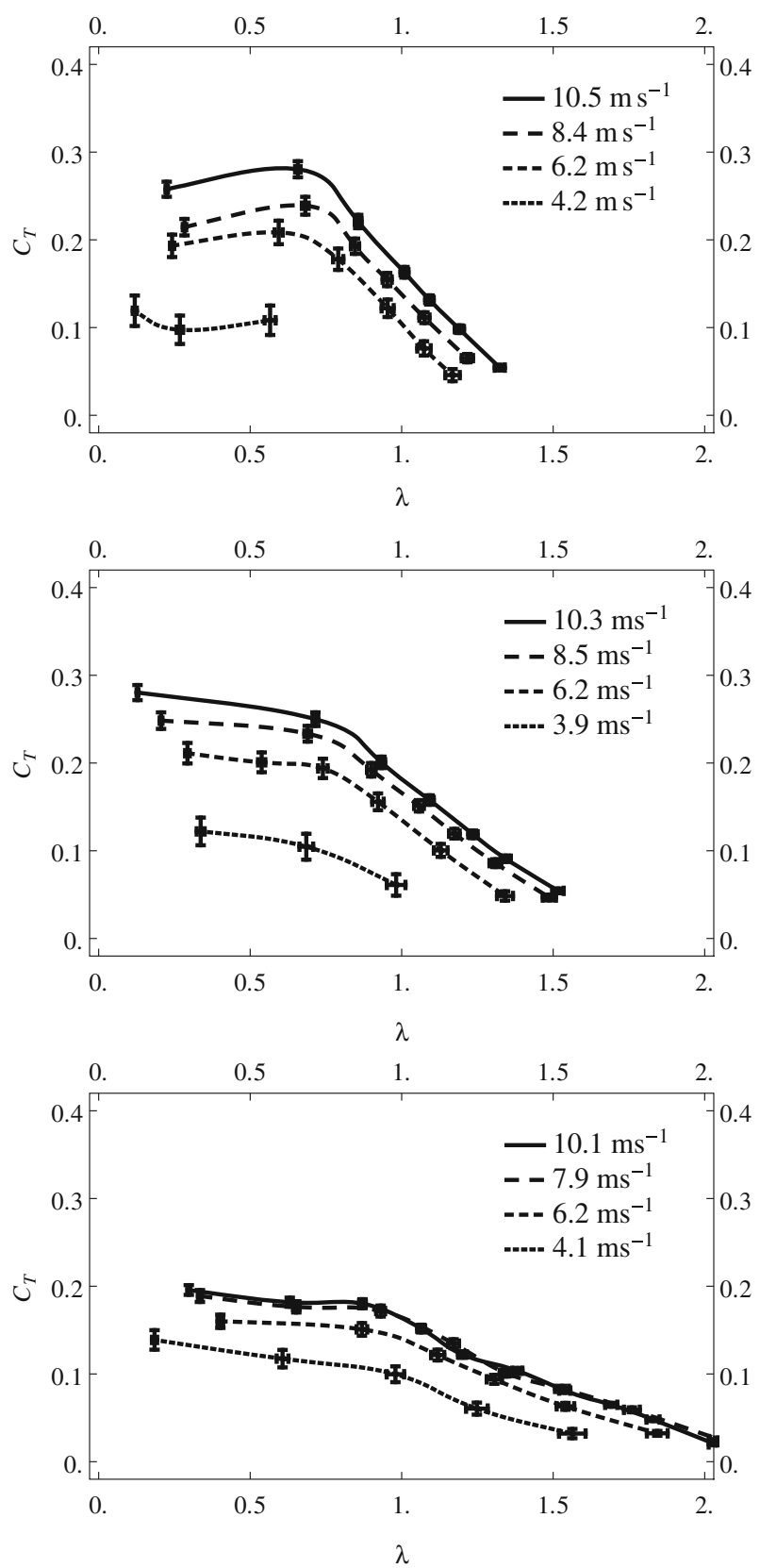

Fig. $5 C_{T}$ distribution as a function of $\lambda$ (Savonius - top, $20^{\circ}-$ middle and $40^{\circ}-$ bottom)

\subsection{Experimental results}

Measurements were carried out in the closed return wind tunnel, shown in Fig. 3. The wind tunnel can attain maximal velocity of about $50 \mathrm{~m} \mathrm{~s}^{-1}$. The wind speed is determined by 


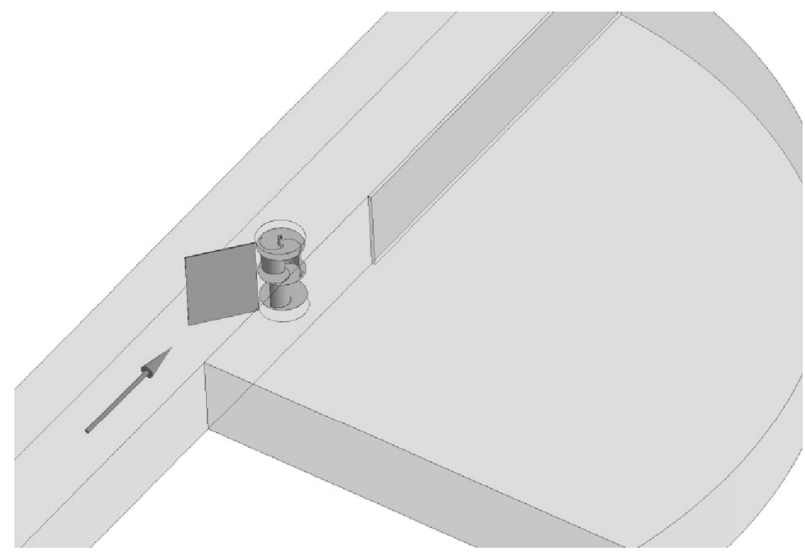

Fig. 6 Flow domain

means of a pitot tube located at the inlet to the test section. The two-stage Savonius design equipped with a stator is located inside the test section with one side of the section open. The cross-sectional area $W \times H_{t}$ is $1.43 \mathrm{~m} \times 0.95 \mathrm{~m}$. The inflow velocity at the inlet to the test section was measured for each case together with the rotational speed of the turbine and the torque acting on the shaft. The tunnel blockage ratio, defined as $H D / H_{t} / W$, is 0.26 for the Savonius design and 0.31, 0.46 for the modified design with the stator angle $20^{\circ}$ and $40^{\circ}$, respectively.

Experimental results of the wind tunnel measurements are shown in Figs. 4 and 5. The former presents the power $C_{P}$ and the latter the torque coefficient $C_{T}$ distributions as a function of tip speed ratios $\lambda$. Absolute errors are shown as an indication of the uncertainty in measurements.

It is clear that the torque coefficient distributions of the modified design are lower in comparison with the original Savonius wind turbine. The larger the stator angle the smaller the values of $C_{T}$ for the corresponding wind speeds. This is not a surprise keeping in mind that the active surface area $H D$ in Eq. 1 is larger for the modified design due to the presence of the stator. What is more, the same stator directing the flow makes it possible to generate higher torques acting on a shaft and to compensate the effect of larger active surface area $H D$ present in Eq. 3. This results in higher efficiencies or the power coefficients $C_{P}$ shown in Fig. 4 and may be regarded as an advantage of the modified design over the Savonius wind turbine. For instance, the highest measured efficiency (3) for the Savonius design is $19 \%$ while for the modified design, with the stator at $20^{\circ}$ and $40^{\circ}$, the highest efficiency is

Table 1 Mesh statistics

\begin{tabular}{llll}
\hline & Savonius & $20^{\circ}$ & $40^{\circ}$ \\
\hline Nodes & 2459557 & 2496403 & 2496106 \\
Elements total & 11082969 & 11171561 & 11162695 \\
Tetrahedral & 9610941 & 9641158 & 9629399 \\
Wedges & 1471022 & 1529400 & 1532288 \\
Pyramids & 1006 & 1003 & 1008 \\
\hline
\end{tabular}




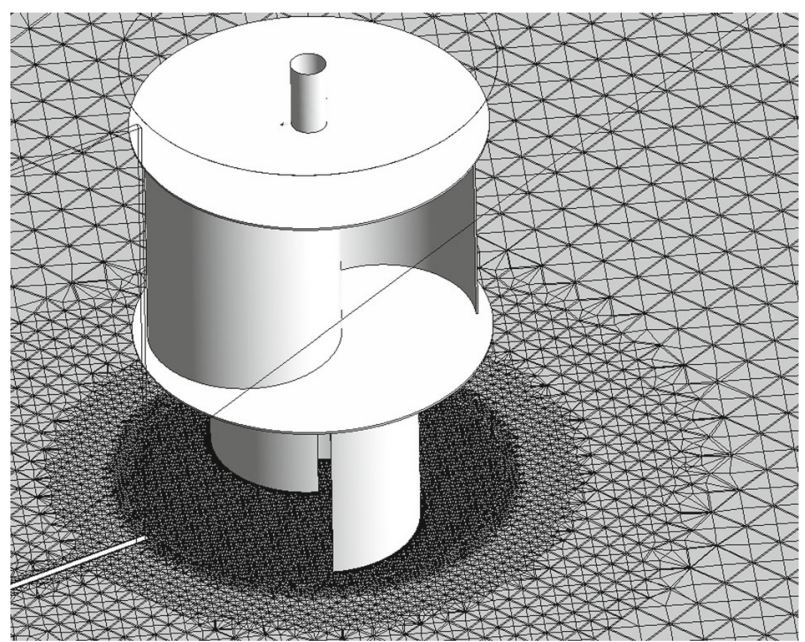

Fig. 7 Mesh cross-section

$23 \%$ and $29 \%$, respectively, for the same wind speed $10 \mathrm{~m} \mathrm{~s}^{-1}$. It is evident that even for low wind speeds the innovative design if more efficient.

\section{Computations}

\subsection{Methodology}

All the numerical calculations have been performed by means of the commercial CFD code CFX. The turbulent flow of air is treated as an incompressible medium. The turbulence is modelled by means of the $k-\omega$ SST (Shear Stress Transport) turbulence model. Preliminary calculations were carried out using the standard $k-\varepsilon$ model. The reason for this choice may be explained by a need of comparison with other calculations

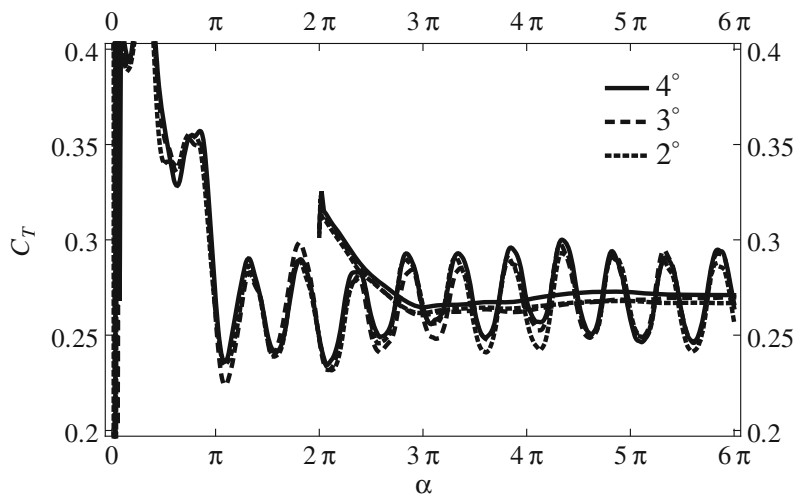

Fig. 8 Time step convergence 
found elsewhere. Both models $k-\varepsilon$ and SST have now become a standard for this kind of calculations. Good applicability of this code for solving flow problems has already been tested. The latter model gives slightly better agreement between computation and experiment.

The governing equations are discretised by means of the finite volume method. The high resolution scheme is used to approximate the advection terms and the second order backward Euler scheme is utilised to approximate the transient terms. Root mean square normalised residual values below $10^{-4}$ per time step are selected as a convergence criteria.

\subsection{Flow domain and boundary conditions}

The flow domain (Fig. 6) is divided into two parts: the rotating rotor and the steady wind tunnel. Both parts are merged by means of the domain interface of the so called 'transient rotor-stator' type. The distance between the interface and the rotor was about $12 \mathrm{~mm}$. The time step of the transient calculations corresponds to four degree of revolution and the rotational speed of the rotating domain (rotor) corresponds to two and three revolutions per second.

The boundary conditions selected here are:

- Inlet. The average velocity is specified here. The turbulence intensity defined as $\tau_{t}=\langle U\rangle^{-1}(2 / 3 k)^{1 / 2}$ equals $5 \%$ and the viscosity ratio $\mu_{t} \mu^{-1}=10$. This represents a medium turbulent intensity.

- Opening. The so called 'far field' condition is chosen with prescribed constant atmospheric pressure.

- Symmetry. This means than the velocity normal component equals zero $\hat{\mathbf{n}} \cdot\langle\mathbf{U}\rangle=0$ and all the scalar values $\varphi$ must fulfil $\hat{\mathbf{n}} \cdot \nabla \varphi=0$ where $\hat{\mathbf{n}}$ represents a unit vector normal to the surface.

- Wall. The flow domain resembles a wind tunnel with one side partially open to the atmosphere, see Figs. 6 and 3. As for the rotor blades, plates and the shaft they are modelled as no slip wall in the rotating frame of reference.

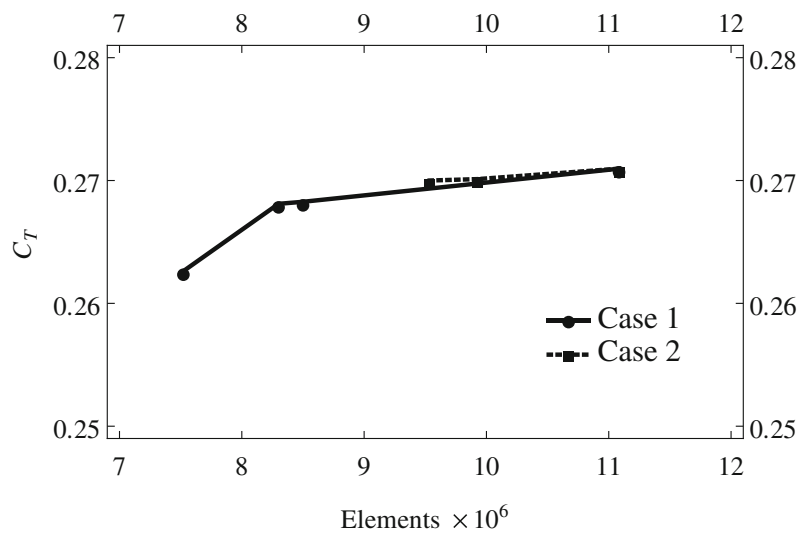

Fig. 9 Mesh convergence 
Fig. $10 C_{T}$ distribution as a function of the angular position of the rotor $\alpha$ (wind speed $6.3 \mathrm{~m} \mathrm{~s}^{-1}$ - top, $8.5 \mathrm{~m} \mathrm{~s}^{-1}$ middle and $10.5 \mathrm{~m} \mathrm{~s}^{-1}$ - bottom)
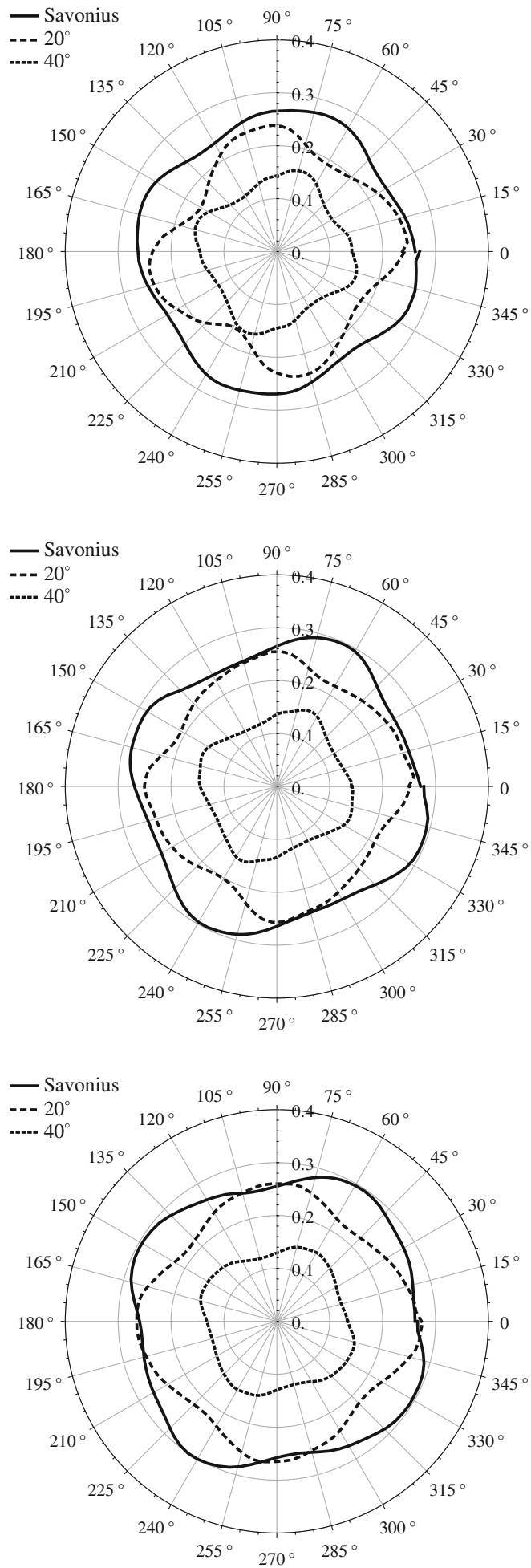
Fig. $11-\mathcal{Q}$ distribution as a function of the angular position of the rotor $\alpha$ (wind speed $6.3 \mathrm{~m} \mathrm{~s}^{-1}$ - top, $8.5 \mathrm{~m} \mathrm{~s}^{-1}$ middle and $10.5 \mathrm{~m} \mathrm{~s}^{-1}$ - bottom)
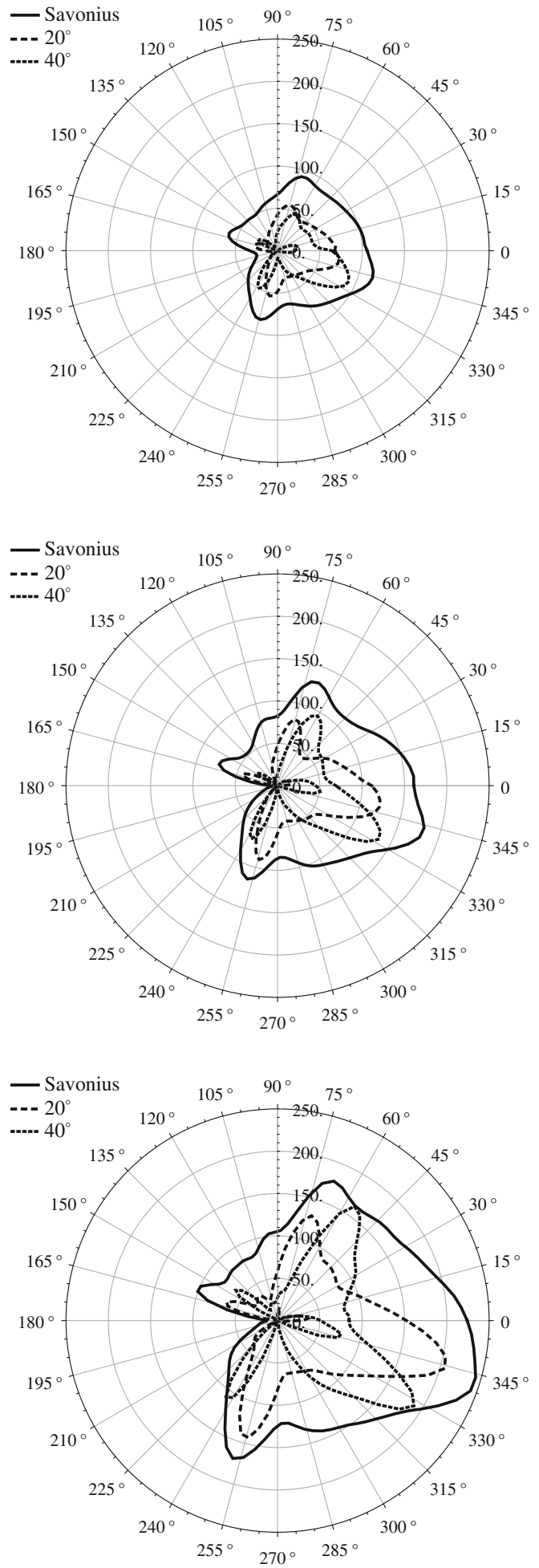
Table 2 Velocity gradient second invariant $\langle Q\rangle /\left\langle Q_{S}\right\rangle$

\begin{tabular}{lllll}
\hline$n\left[\mathrm{rev} \mathrm{s}^{-1}\right]$ & $U\left[\mathrm{~m} \mathrm{~s}^{-1}\right]$ & Savonius & $20^{\circ}$ & $40^{\circ}$ \\
\hline 2 & 6.3 & 0.433 & 0.244 & 0.173 \\
& 8.5 & 0.722 & 0.422 & 0.354 \\
& 10.5 & 1.000 & 0.534 & 0.322 \\
3 & 6.3 & 0.551 & 0.281 & 0.217 \\
& 8.5 & 0.724 & 0.347 & 0.266 \\
& 10.5 & 1.000 & 0.573 & 0.404 \\
\hline
\end{tabular}

\subsection{Mesh}

Both flow domains, i.e. the rotor and the wind tunnel, were discretised separately. Both domains have an unstructured grid consisting of mostly tetrahedral elements. The total number of elements covering the flow area is above 11 million. There are also special elements around the blades to ensure that flow near a wall is properly resolved. The scalable wall function approach has been used to provide near wall boundary conditions for the mean flow. The quality of the grid near the blades may be inspected in terms of the average value of $y^{+}$distribution

$$
\bar{y}^{+}=\frac{1}{|S|} \iint_{S} y^{+} \mathrm{d} S
$$

where $|S|$ stands for the area of blade surface $S$. The average $\bar{y}^{+}$value was in the range $1-2$ and the maximal 5-6 for all angles.

Table 1 shows the mesh statistics for the considered flow geometries. Figure 6 presents global view on the computational domain that consists of the wind tunnel (see Fig. 3) and a quarter-cylindrical part of 'far field' surroundings. The mesh can be inspected in Fig. 7. This figure presents a cross-section which is perpendicular to the rotor axis.

A time step convergence for the Savonius design $\left(n=3 \mathrm{rev} \mathrm{s}^{-1}, U=8.5 \mathrm{~m} \mathrm{~s}^{-1}\right)$ for three revolutions is shown in Fig. 8. Three different case are presented, namely $4^{\circ}, 3^{\circ}$ and $2^{\circ}$ per time-step. Additionally, moving averages of the torque coefficient $C_{T}$ are superimposed. It is evident that decreasing the temporal resolution from $4^{\circ}$ to $2^{\circ}$ per time-step has little effect on the results in terms of $C_{T}$. However, it makes it possible to increase

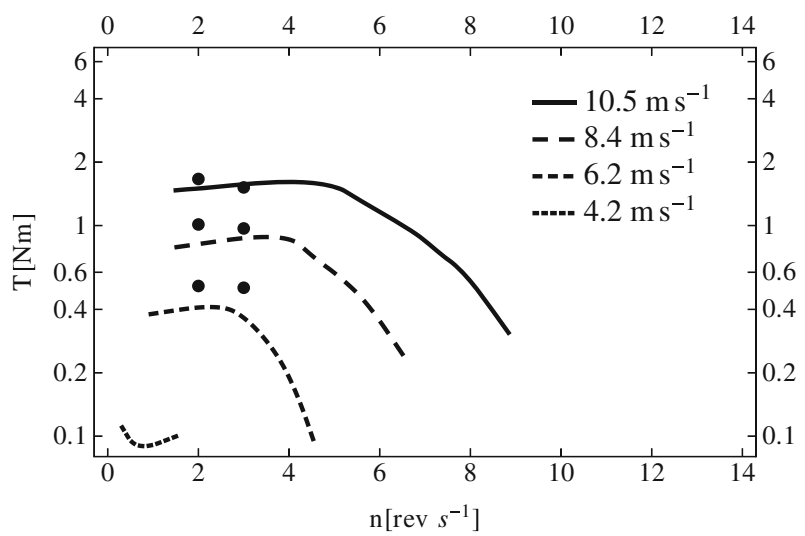

Fig. 12 Torque $T$ distribution as a function of the rotational speed $n$ for the Savonius wind turbine 
the computational speed. In general, the transient simulations requires typically 3 to 5 revolutions to reach periodic state.

Figure 9 demonstrates mesh convergence by showing the influence of number of elements on the torque coefficient $C_{T}$. Two cases are considered here. By 'Case 1' it is meant the increase in the boundary layer elements and decrease in this layer thickness. Special care needs to be taken around the blades and plates where the velocity gradients are very high. Increasing the number of the mesh elements above $10 \times 10^{6}$ has negligible effects on the torque coefficient $C_{T}$. By 'Case 2' it is meant the increase in the rotor vicinity mesh keeping the boundary layer elements and its thickness constant and properly resolved. One can observe little influence of the mesh size on $C_{T}$. Results are nearly constant within the range of $9.5-11 \times 10^{6}$ elements.

\subsection{Evaluation and comparison criteria}

These criteria allow for direct evaluation and comparison of various designs and solutions. One should formulate criteria that are invariant. This is because they remain unchanged under certain transformation. Invariants of velocity gradient tensors are used in turbulence modelling because they contain all the necessary information involving the rates of rotation, stretching and angular deformation being responsible for kinetic energy dissipation and vortex stretching [17]. Invariants of velocity gradient tensor and their analysis are useful because they unambiguously determine the local topology of the fluid motion [4].

Let us first recall enstrophy $\mathcal{E}^{*}[17]$

$$
\mathcal{E}^{*}=\iiint_{V}\|\mathbf{A}\|^{2} \mathrm{~d} V=\iiint_{V} \frac{1}{2}\|\boldsymbol{\Omega}\|^{2} \mathrm{~d} V=\iiint_{V} \epsilon^{*} \mathrm{~d} V,
$$

where $\epsilon^{*}=2^{-1}\|\boldsymbol{\Omega}\|^{2}$ may be regarded as a specific enstrophy. Here $\mathbf{A}$ stands for asymmetrical part of the velocity gradient tensor $\nabla \mathbf{U}$ and $\boldsymbol{\Omega}$ is the vorticity vector. Enstrophy determines the rate of dissipation of kinetic energy being a global measure of the dissipation rate and vorticity measure.

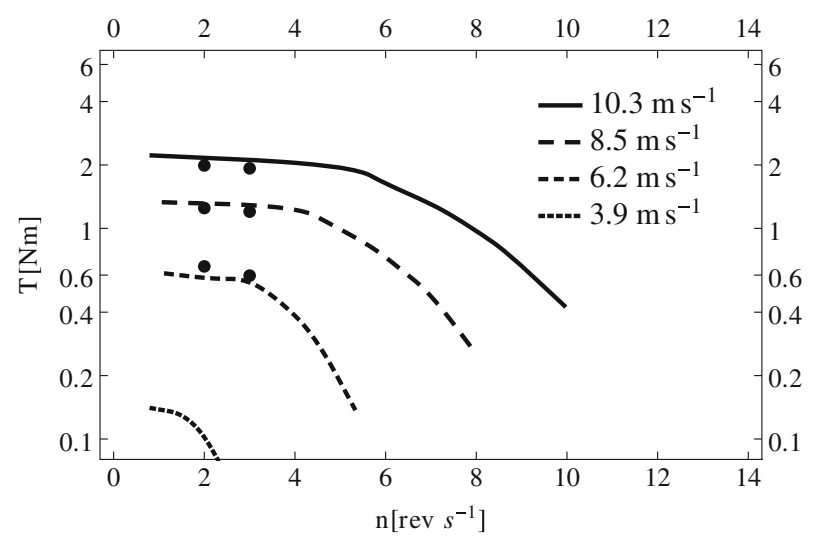

Fig. 13 Torque $T$ distribution as a function of the rotational speed $n$ for the modified wind turbine $\left(20^{\circ}\right)$ 
The vorticity measure $\mathcal{Q}$, investigated further, is defined by means of the integral of the velocity gradient tensor second invariant in the following form

$$
\mathcal{Q}=\iiint_{V} \operatorname{tr}(\nabla \mathbf{U})^{2} \mathrm{~d} V=\iiint_{V}\left(\operatorname{tr} \mathbf{D}^{2}-\epsilon^{*}\right) \mathrm{d} V,
$$

where $\mathbf{D}$ stands for symmetrical part of the velocity gradient tensor. Bearing in mind the definition of the dissipation function $\phi_{\mu}=2 \mu \operatorname{tr} \mathbf{D}^{2}$ and the dissipation power $P_{d}=$ $\iiint_{V} \phi_{\mu} \mathrm{d} V$ as well as enstrophy defined in Eq. 6 we arrive at

$$
\mathcal{Q}=\frac{P_{d}}{2 \mu}-\mathcal{E}^{*}
$$

The above criterion represents a global balance between the power of energy dissipation and vortex structures by means of the vorticity magnitude or enstrophy. These structures are generated by the rotor and may affect the efficiency. Equation 8 is appropriate for each time step, while for the total revolution one should use the time integral of $\mathcal{Q}$ instead

$$
\langle\mathcal{Q}\rangle=\frac{1}{\Delta t} \int_{t}^{t+\Delta t} \mathcal{Q} \mathrm{d} t .
$$

\subsection{Results}

Figure 10 present the torque coefficient $C_{T}$ distribution as a function of revolution angle $\alpha$ for various wind speeds and for $n=3 \mathrm{rev} \mathrm{s}^{-1}$. The present calculations are fully transient and three-dimensional. The four peaks are visible in the curves instead of two. This is because the device consists of two rotors rotated by an angle relative to each other. Additionally, there is a stator directing the flow. The presence of the stator increases the active surface area $H D$. As in the previous case (Fig. 5), the torque coefficient distributions of the modified design are lower in comparison with the original Savonius wind turbine.

Figure 11 shows the distribution of the vorticity measure $-\mathcal{Q}$ defined by means of Eqs. 7 or 8 which can be used to compare various designs. This indicates the importance of this equation because it makes it possible to visualise the global balance between the power of

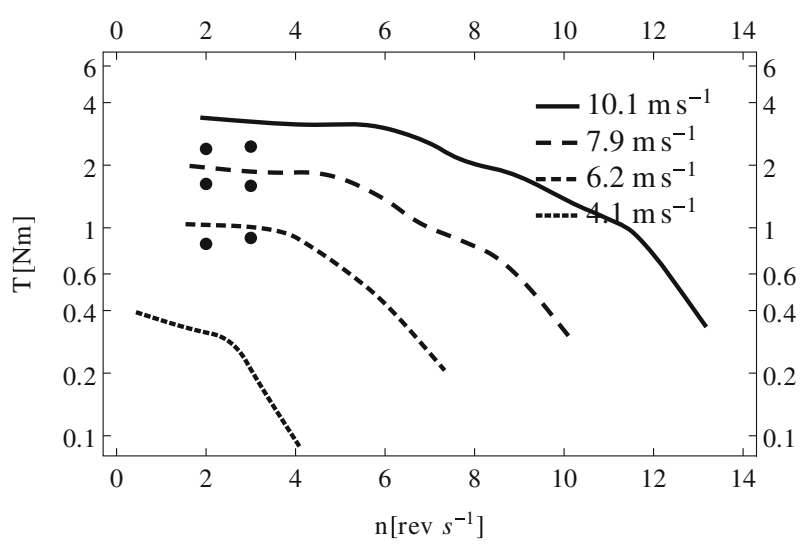

Fig. 14 Torque $T$ distribution as a function of the rotational speed $n$ for the modified wind turbine $\left(40^{\circ}\right)$ 
energy dissipation and enstrophy as a function of the angular position $\alpha$ of the rotor. It is visible that the more efficient the turbine the lower the values of $-\mathcal{Q}$. The time integral (9) of this measure can be used directly to evaluate specific turbine in terms of a vorticity measure. Table 2 presents the ratio of $\langle Q\rangle$ to $\left\langle Q_{S}\right\rangle$ of the respective Savonius design. It simply confirms previous observations. Furthermore, one can anticipate a relationship between vorticity measure and efficiency of a turbine. It can be expected that the performance of a wind turbine is affected by vortices generated during the rotor revolutions. These vortices are complicated in their nature and dynamically change their configurations and undoubtedly intensities. This may affect pressure distribution around the blades and accordingly the performance of a rotor.

Figures 12-14 show the measured torques $T$ versus CFD calculations for the three designs as a function of rotational speed $n$. Black dots correspond to the numerical prediction. The above comparison should be used as a reference point. This is because the velocities for the CFD calculations were kept at the same level for the different designs.

\section{Conclusions}

A three-dimensional analysis of the transient aerodynamic phenomena occurring in the innovative modification of the classic Savonius wind turbine has been shown. This includes both: transient CFD calculations and wind tunnel experiments both being consistent. The innovative modification of the classic Savonius design shows higher efficiency. This is due to presence of the stator which directs the air and generate higher torques acting on a shaft. Furthermore, it makes it possible to take better advantage of wind energy and compensate the effect of larger active surface area. The larger the stator angle the higher the efficiency. This is true for wind tunnel experiments at least.

A vorticity measure, based on the integral of the velocity gradient tensor second invariant, has been proposed allowing for evaluation and comparison of various designs. The proposed quantity represents a global measure between the dissipated power and enstrophy. The latter is related to vortex structures through the vorticity magnitude. The time integral of this measures can be used directly to evaluate specific turbine. A relationship between the proposed vorticity measure and efficiency of a turbine can be anticipated. The higher the efficiency the lower the the time integral of $-\mathcal{Q}$. It is then expected that vortex structures generated by the rotor affect pressure distribution around the blades and accordingly the performance of a rotor.

\section{References}

1. Afungchui, D., Kamoun, B., Helali, A., Djemaa, A.B.: The unsteady pressure field and the aerodynamic performances of Savonius rotor based on the discrete vortex method. Renew. Energy 35, 307-313 (2010)

2. Altan, B.D., Atilgan, M., Ozdamar, A.: An experimental study on improvement of a Savonius rotor performance with curtaining. Exp. Thermal Fluid Sci. 32, 1673-1678 (2008)

3. Blackwell, B.F., Sheldahl, R.E., Feltz, L.V.: Wind tunnel performance data for two- and three-bucket Savonius rotor. Sandia Laboratories Report SAND 76-0131 (1977). http://prod.sandia.gov/techlib/ accesscontrol.cgi/1976/760131.pdf

4. Chong, M.S., Perry, A.E., Cantwell, B.J.: A general classification of threedimensional flow fields. Phys. Fluids A 2, 765-777 (1990)

5. Doerffer, P.: International patent application PCT/PL2012/000125 and national patent application

6. Fujisawa, N., Gotoh F.: Visualisation study of the flow in and around a Savonius rotor. Exp. Fluids 12, 407-412 (1992) 
7. Kamoji, M.A., Kedare, S.B., Prabhu, S.V.: Experimental investigations on single stage, two stage and three stage conventional Savonius rotor. Int. J. Energy Res. 32, 877-895 (2008)

8. Kludzinska, K., Swirydczuk, J.: Unsteady flow through the gap of Savonius turbine rotor. European congress on computational methods in applied sciences and engineering (ECCOMAS 2012), Vienna, Austria (2012)

9. Kludzinska, K., Swirydczuk, J.: A numerical analysis of the unsteady flow round a Savonius turbine. XX polish fluid mechanics conference, Gliwice (2012)

10. Menet, J.: Prediction of the aerodynamics of a new type of vertical axis wind turbine: the reverse bladed rotor. In: Proceedings of the european wind energy conference (2008)

11. Mohamed, M.H., Janiga, G., Pap, E., Thevenin D.: Optimisation of Savonius turbines using an obstacle shielding the returning blade. Renew. Energy 35, 2618-2626 (2010)

12. Nakajima, M., Iio, S., Ikeda, T.: Performance of double-step Savonius rotor for environmentally friendly hydraulic turbine. J. Fluid Sci. Technol. 3, 410-419 (2008)

13. Ogawa T.: Theoretical study on the flow about Savonius rotor. ASME J. Fluids Eng. 106, 85-91 (1984)

14. Saha, U.K., Thotla, S., Maity D.: Optimum design configuration of Savonius rotor through wind tunnel experiments. J. Wind Eng. Ind. Aerodyn. 96, 1359-1375 (2008)

15. Swirydczuk, J., Doerffer, P., Szymaniak, M.: Unsteady flow through the gap of Savonius turbine rotor. Task Quarterly 15(1), 59-70 (2011)

16. Swirydczuk, J., Kludzinska, K.: Improving Savonius rotor performance by shaping its blade edges. Herald of aeroenginebuilding, No 2, pp. 86-90, ISSN 1727-0219 (2012)

17. Tesch, K.: On invariants of fluid mechanics tensors. Task Quarterly 17(3-4), 1000-1008 (2013) 